random group. Separate studies partitioning each of the conditions may pull out the contributing factors to these effects.

The main findings of this study were that "certainty" and "uncertainty" about the time to expect shock does affect the anticipatory cardiac response. Furthermore, different cue inputs can serve as a mediating variable affecting this response. Amplified cardiac cues increased the "modulation" of heart-rate responses, while external cues of less relevancy tended to interfere with the "spontaneous" heart-rate activity.

A light signalling the occurrence of a shock at some future time leads to heart-rate acceleration, perhaps as a "fear" reaction but more wikely as a general preparatory response. The steepest gradients of heart-rate acceleration in response to the signal lead reliably to gradients of deceleration in an interval prior to shock, and to the least steep gradients of acceleration in response to shock. Conversely, the least steep gradients of signal acceleration lead reliably to gradients of acceleration in an interval prior to shock, and to the greater magnitude acceleration in response to shock.

\section{REFERENCES}

DEANE, G. E. Human heart rate responses during experimentally induced anxiety. Journal of Experimental Psychology, 1961, 6, 489-493.

ELLIOTT, R. Effects of uncertainty upon the nature and advent of a noxious stimulus (shock) upon heart rate. Journal of Personality \& Social Psychology, 1966, 3, 353-356.

FENZ, W. D. Specificity in somatic response to anxiety. Perceptual \& Motor Skills, 1967, 24, 1183-1190.

FENZ, W. D., \& EPSTEIN, S. Manifest anxiety: Unifactorial or multifactorial composition? Perceptual \& Motor Skills, 1965, 20, 773-780.

JONES, A. Information deprivation in humans. In B. A. Maher (Ed.), Progress in experimental personality research. New York: Academic Press, 1966. Pp. 241-315.

OBRIST, P. Heart rate during classical conditioning in humans and dogs: Significance for psychological processes. In I. Ruttkay-Nedecky, L. Ciganek, V. Zikmund, and E. Kellerova (Eds.), Mechanisms of orienting reaction in man. Bratislava: Slovak Academy of Sciences, 1967. (Transactions of an international colloquium held in Bratislava and Smolenice, 1965, pp. 11-14, under the auspices of the Slovak Academy of Sciences.)

OBRIST, P. Heart rate and somatic-motor coupling during classical aversive conditioning in humans. Journal of Experimental Psychology, 1968, 75, 000-000.

PERVIN, L. A. The need to predict and control under conditions of threat. Journal of Personality, 1963, 31, 570-587.

WILSON, R. S., \& DUERFELDT, P. H. Cardiac responsiveness and differential conditioning. Journal of Comparative \& Physiological Psychology, 1967,63, 87-94.

ZEAMAN, D., \& SMITH, R. W. Human cardiac conditioning. In W. F. Prokasy (Ed.), Classical conditioning: $A$ symposium. New York: Appleton-Century-Crofts, 1965. Pp. 378-418. NOTE

1. This report is based on a thesis submitted by Alvin H. Shapiro in partial fulfillment of the requirements for an MA degree. It was presented at the annual meeting of the Society for Research in Psychophysiology, Washington, D.C., October
1968. The research was supported by Grant MA 2330 from the Medical Research Council of Canada to Walter D. Fenz.

\title{
Differential training in multiple probability-learning and decision-making
}

LOWELL M. SCHIPPER, Pennsylvania State University, University Park, Pa. 16802

Differential training in a multiple probability-learning situation, with two of the three probabilities receiving, respectively, 60-60, 60-180, 180-60, 180-180 training trials, showed nonsymmetric effects of this training when the two differentially trained probabilities were $\pi_{1}=1 / 6$ and $\pi_{3}=5 / 6 . \quad \pi_{2}=.50$ received a constant 120 training trials for all four treatments.

The purpose of this study was to investigate the use of cues which had received different amounts of training in a multiple probability learning situation in a subsequent decision-making task where combinations of cues were presented. Earlier studies (Schipper, 1967, 1966a, b) have looked at similar tasks but only with equivalent training for all cues.

\section{METHOD}

Subjects received training trials on each of three predictor (green) lights in a multiple probability learning situation. On each trial one of these lights (arranged linearly and

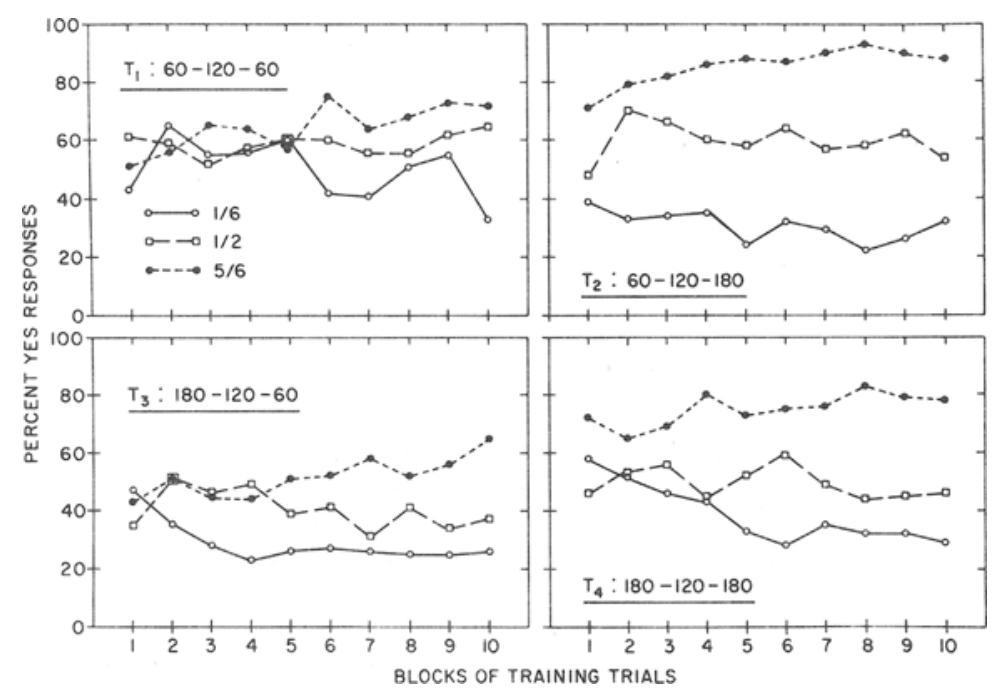

Fig. 1. Per cent yes responses on individual predictors in training. horizontally) came on for $3 \frac{1 / 2}{\mathrm{sec}}$, after which an event (red) light either came on with the green light for an additional 1 sec or did not come on with respective probabilities of $1 / 6,1 / 2$, and $5 / 6$. The intertrial interval was 2 sec.

Differential training on the three green lights was assigned according to the following schedule: $T_{1}(\mathrm{~N}=16)-\pi_{1}=1 / 6$, 60 trials; $\pi_{2}=1 / 2,120$ trials; $\pi_{3}=5 / 6,60$ trials. $T_{2}(N=18)-\pi_{1}=1 / 6,60$ trials; $\pi_{2}=1 / 2,120$ trials; $\pi_{3}=5 / 6,180$ trials. $T_{3}$ $(\mathrm{N}=17)-\pi_{1}=1 / 6,180$ trials; $\pi_{2}=1 / 2$, 120 trials; $\pi_{3}=5 / 6, \quad 60$ trials. $T_{4}$ $(N=18)-\pi_{1}=1 / 6,180$ trials; $\pi_{2}=1 / 2$, 120 trials; $\pi_{3}=5 / 6,180$ trials. The experiment can be thought of as a simple 2 by 2 factorial design with two training levels of the 1/6 light and two training levels of the 5/6 light arranged orthogonally. Training with the $1 / 2$ light is constant.

On each trial Ss predicted whether or not the red light would occur following the particular green light. Responses were made on a Digitek answer sheet using one column for "yes" responses and another column for "no" responses. All Ss were volunteers from introductory psychology at PSU and were assigned to the treatments without bias. 
Table 1

Proportion Yes Responses in Decision-Making

\begin{tabular}{|c|c|c|c|c|c|c|c|c|c|}
\hline & $1 / 6$ & $1 / 2$ & $5 / 6$ & $1 / 6-1 / 2$ & $1 / 6-5 / 6$ & $1 / 2-5 / 6$ & $1 / 6-1 / 2-5 / 6$ & A.E. $1 / 6$ & A.E. $5 / 6$ \\
\hline $\begin{array}{l}\text { Treatment } 1 \\
\text { Treatment } 2 \\
\text { Treatment } 3 \\
\text { Treatment } 4\end{array}$ & $\begin{array}{l}.306 \\
.149 \\
.211 \\
.305\end{array}$ & $\begin{array}{l}.643 \\
.638 \\
.411 \\
.366\end{array}$ & $\begin{array}{l}.681 \\
.872 \\
.664 \\
.733\end{array}$ & $\begin{array}{l}.525 \\
.344 \\
.276 \\
.411\end{array}$ & $\begin{array}{l}.493 \\
.555 \\
.388 \\
.666\end{array}$ & $\begin{array}{l}.693 \\
.933 \\
.676 \\
.722\end{array}$ & $\begin{array}{r}.781 \\
.794 \\
.676 \\
.672\end{array}$ & $\begin{array}{l}-.153 \\
-.305 \\
-.205 \\
-.011\end{array}$ & $\begin{array}{l}+.118 \\
+.350 \\
+.221 \\
+.385\end{array}$ \\
\hline
\end{tabular}

Number of Ss Making 0 through 10 Yes Responses for Multiple Light Patterns

\begin{tabular}{rrrrr}
10 & 5 & 8 & 23 & 23 \\
9 & 2 & 4 & 12 & 13 \\
8 & 4 & 9 & 9 & 6 \\
7 & 7 & 5 & 5 & 4 \\
6 & 4 & 3 & 3 & 6 \\
5 & 6 & 12 & 7 & 4 \\
4 & 2 & 9 & 4 & 2 \\
3 & 5 & 7 & 3 & 2 \\
2 & 13 & 2 & 0 & 4 \\
1 & 15 & 3 & 1 & 3 \\
0 & 6 & 7 & 2 & 2 \\
\hline
\end{tabular}

After the training trials, all Ss were given 10 presentations of each of the seven possible combinations of probabilities in random arrangements of blocks of seven trials. In this latter part of the experiment, the red light was covered and $S$ received no feedback concerning the appropriateness of a yes or no response.

\section{RESULTS}

The learning data are summarized in Figs. 1a through $1 \mathrm{~d}$. The overall block sizes for the four groups differ because of the different amounts of training for each of the presented predictor lights in the DM trials. This can be examined by looking at the $1 / 6-1 / 2,1 / 6-5 / 6,1 / 2-5 / 6$, and $1 / 6-1 / 2-5 / 6$ patterns. For this purpose it is particularly useful to consider the design as a 2 by 2 arrangement with the four cells of the table representing the combinations of training trials for the two extreme predictors. Four separate analyses can be carried out, one for each of the combinations of predictors. These analyses are summarized in Table 2 with $F$ ratios calculated in the traditional manner ( $1 \mathrm{df}$ in the numerator, and $65 \mathrm{df}$ in the denominator for each of the Fs).

Table 2

\begin{tabular}{llcccc}
\hline & & $1 / 6-1 / 2$ & $1 / 6-5 / 6$ & $1 / 2-5 / 6$ & $1 / 6-1 / 2-5 / 6$ \\
\hline & & $F$ & $F$ & $F$ & F \\
Treatments $1 \& 2$ vs 3 \& 4 & Lo 1/6 vs Hi 1/6 & 1.286 & $<1$ & 2.844 & 2.434 \\
Treatments 1 \& 3 vs 2 \& 4 & Lo 5/6 vs Hi 5/6 & $<1$ & 5.798 & 3.989 & $<1$ \\
Treatments 1 \& 4 vs 2 \& 3 & Interaction & 4.562 & 2.312 & 1.732 & $<1$ \\
\hline
\end{tabular}

predictors. Thus, in Fig. 1a each complete block is made up of 24 trials, 6 with the $\pi=1 / 6$ predictor, 12 with the $\pi=1 / 2$ predictor, and 6 with the $\pi=5 / 6$ predictor randomly arranged. In Figs. $1 \mathrm{~b}$ and $1 \mathrm{c}$ each complete block is made up of $6+12+18$ and $18+12+6=36$ trials, and in Fig. 1d each block is made up of $18+12+18=48$ trials. Some of the instability of these curves, therefore, is attributable to different numbers of trials in blocks on which the respective points are based. Within each block, of course, the respective $\pi$ values are preserved.

Table 1 shows the percentages of predictions of the red light following each pattern in the decision-making trials. Each entry is based on 10 trials per $S$. The distribution of predictions on a per-S basis is shown in the bottom half of the table.

\section{DISCUSSION}

Of prime interest is the way Ss combined the information from the separately
It can be seen that the 60 vs 180 training trials for the $\pi=1 / 6$ predictor produced no significant effect for any of the DM patterns. Changing from 60 to 180 trials for the $\pi=5 / 6$ predictor did, however, produce two marked response changes in predictions of the event light. The actual differences can be obtained by comparing Treatments 1 and 3 , together, with Treatments 2 and 4, together, in Table 1 for the $1 / 6-5 / 6$ pattern (Diff. $=.17, p<.02$ ) and for the $1 / 2-5 / 6$ pattern (Diff. $=.14, \quad \mathrm{p}=.05$ ). These differences in proportions for predicting the event light are both in favor of more predictions for the groups with more training on the $\pi=5 / 6$ predictor. The statistical difference in the $1 / 6-5 / 6$ pattern need not be qualified by the shape of the distribution of the proportions of responses, but the differences in the $1 / 2-5 / 6$ pattern probably should be. The distribution here is skewed negatively with a mean of 7.59 and a variance of 7.08 .
The difference between Treatments 1 and 4 and Treatments 2 and 3 for the 1/6-1/2 combination $(F=4.562, \quad p<.02)$ is difficult to interpret since it represents a pooling of 60 and 180 training trials for both combinations of treatments. One possible reason for the difference might be the relatively high proportions of predictions of the single event light following $\pi=1 / 6$ for Treatments 1 and 4 (.306 and .305) compared with the corresponding proportions for Treatments 2 and 3 (.149 and .211).

Another way to interpret the use of the differential training is to measure the average effect of adding one predictor to another (Schipper, 1967). Using this procedure, the average effect of the 1/6 predictor can be calculated by observing its effect when comparing the $1 / 6-1 / 2$ and $1 / 6-5 / 6$ combinations with the single $1 / 2$ and $5 / 6$ presentations. Thus, [(.643 -.525$)$ $+(.681-.493)] / 2=.153$, which is the average reduction by the $1 / 6$ predictor when added to the separate independent $1 / 2$ and $5 / 6$ predictors. These average effects for the $\pi=1 / 6$ and $\pi=5 / 6$ predictors are shown in Table 1 in the two right-hand columns. Of interest is the importance of the increased effect of greater training with the $\pi=5 / 6$ predictor as compared with the corresponding effect of the $\pi=1 / 6$ predictor. This asymmetry implies that equivalent training on similar cues results in quite different subsequent use of the cues in a more complex prediction situation.

\section{REFERENCES}

SCHIPPER, L. M. Extreme probabilities in learning and decision making. Journal of Experimental Psychology, 1967, 73, 149-151. SCHIPPER, L. M. Context effects in probability learning and decision-making. Psychological Reports, 1966a, 18, 131-138.

GENTILE, J. R., \& SCHIPPER, L. M. Personality variables in probability-learning, decision-making, and risk-taking. Perceptual \& Motor Skills, 1966b, 22, 583-591. 